\title{
An innovative approach to predict immune-associated genes mutually targeted by cow and human milk microRNAs expression profiles
}

\author{
Kaj Chokeshaiusaha1§, Thanida Sananmuang ${ }^{1 \S}$, Denis Puthier ${ }^{2}$ and Catherine Nguyen²
}

1. Department of Veterinary Science, Faculty of Veterinary Medicine, Rajamangala University of Technology Tawan-OK, Chonburi, Thailand; 2. Aix-Marseille Université, INSERM UMR 1090, TAGC, Marseille, France. sBoth authors contributed equally to this work.

Corresponding author: Kaj Chokeshaiusaha, e-mail: kaj.chk@gmail.com

Co-authors: TS: tsananmuang@gmail.com, DP: denis.puthier@univ-amu.fr, CN: catherine.nguyen@univ-amu.fr Received: 31-05-2018, Accepted: 16-07-2018, Published online: 01-09-2018

doi: 10.14202/vetworld.2018.1203-1209 How to cite this article: Chokeshaiusaha K, Sananmuang T, Puthier D, Nguyen C (2018) An innovative approach to predict immune-associated genes mutually targeted by cow and human milk microRNAs (miRNAs) expression profiles, Veterinary World, 11(9): 1203-1209.

\begin{abstract}
Aim: Milk is rich in miRNAs - the endogenous small non-coding RNA responsible for gene post-transcriptional silencing. Milk miRNAs were previously evidenced to affect consumer's immune response. While most studies relied on a few wellcharacterized milk miRNAs to relate their immunoregulatory roles on target genes among mammals, this study introduced a procedure to predict the target genes based on overall milk miRNA expression profiles - the miRNome data of cow and human.
\end{abstract}

Materials and Methods: Cow and human milk miRNome expression datasets of cow and human milk lipids at 2, 4, and 6 months of lactation periods were preprocessed and predicted for their target genes using TargetScanHuman. Enrichment analysis was performed using target genes to extract the immune-associated gene ontology (GO) terms shared between the two species. The genes within these terms with more than 50 different miRNAs of each species targeting were selected and reviewed for their immunological functions.

Results: A total of 146 and 129 miRNAs were identified in cow and human milk with several miRNAs reproduced from other previous reports. Enrichment analysis revealed nine immune-related GO terms shared between cow and human (adjusted $\mathrm{p} \leq 0.01$ ). There were 14 genes related to these terms with more than 50 miRNA genes of each species targeting them. These genes were evidenced for their major roles in lymphocyte stimulation and differentiation.

Conclusion: A novel procedure to determine mutual immune-associated genes targeted by milk miRNAs was demonstrated using cow and human milk miRNome data. As far as we know, this was the $1^{\text {st }}$ time that milk miRNA target genes had been identified based on such cross-species approach. Hopefully, the introduced strategy should hereby facilitate a variety of cross-species miRNA studies in the future.

Keywords: immune-associated target gene, microRNAs, milk, miRNome.

\section{Introduction}

Milk contains essential nutrients and bioactive components distributed among its three fractions - lipid, whey, and cell. Besides, milk is also an enriched source of non-coding RNAs, especially the microRNAs (miRNAs) which were verified to be absorbed by various consumers' cells [1-5]. miRNA is an endogenous small non-coding RNA ( $\approx 22$ nucleotides) responsible for silencing its target messenger RNAs (mRNAs) by post-transcriptional degradation [6] and thus is involved in a variety of cell biological processes [7]. In several breastfeeding animals, mammary gland tissues evidently sequestrate remarkable numbers of miRNAs into milk which, in turn, can modulate particular cell mechanisms

Copyright: Chokeshaiusaha, et al. Open Access. This article is distributed under the terms of the Creative Commons Attribution 4.0 International License (http://creativecommons.org/licenses/ by/4.0/), which permits unrestricted use, distribution, and reproduction in any medium, provided you give appropriate credit to the original author(s) and the source, provide a link to the Creative Commons license, and indicate if changes were made. The Creative Commons Public Domain Dedication waiver (http:// creativecommons.org/publicdomain/zero/1.0/) applies to the data made available in this article, unless otherwise stated. of neonate [1-3,5]. Among the whole miRNA spectrum presented - the miRNome expression profiles achieved in milk, the miRNome of milk lipid fraction was regarded to be the best representative for that of mammary gland $[1,2]$.

Equivalent biological influences of milk miRNAs among breastfeeding species were implied by several studies [1-5]. Furthermore, cross-species milk miRNA absorption between human and another breastfeeding mammal was even demonstrated $[8,9]$. Considering such mutual effects of milk miRNAs, the insight into such conserved orthologous genes should render us with novel knowledge for both animal evolution and pediatric sciences. Since milk miRNome analyses are most conventional among cow and human miRNA studies, miRNome expression profiles of these two species are hence considerably available in the public database - making the cross-species analyses between them priorly suitable and profitable.

Milk miRNAs significantly contributed their roles in neonatal immune system $[6,10]$. Human milk miRNAs were evidenced to manifest their effects on T-cell function $[6,11,12]$ and B-cell differentiation [6]. 
Similar immunoregulatory effects were also implied by cow milk miRNAs [10] with reported cross-species effects on human leukocytes $[8,9]$. These evidence strongly suggested the immune-associated genes mutually targeted by both cow and human milk miRNAs and therefore manifested the conserved immunomodulatory effects of milk miRNAs between the two species. By implying such knowledge to other breastfeeding species, identification of these target genes could beneficially provide us with precious clues into fundamental features of miRNA-mRNA interactions across mammalian evolution $[13,14]$.

Due to the aforementioned necessity, we, therefore, demonstrated a cross-species analytical procedure to predict immune-associated genes mutually regulated by both cow and human milk miRNAs. Based on the cumulative number of miRNAs targeting the genes, the mutual immune-associated genes - functioning in lymphocyte development and resting cell state were successfully demonstrated.

\section{Materials and Methods}

Ethical approval

All datasets used in this study were available in NCBI SRA public site, and no ethical approval was required.

\section{Sample datasets}

Cow and human milk miRNome expression datasets were acquired from milk lipids of nursing mothers and dairy cows at approximately 2,4 , and 6 months of lactation periods. All cow and human milk miRNome expression datasets with their corresponding sample filename IDs used in this study were retrieved from the sequence read archive (SRA) database (https://www.ncbi.nlm.nih.gov/sra) (Table-1). Further details of each sample file could be attained from SRA database.

\section{Data preprocessing}

The miRNome expression datasets were extracted and checked for sequencing quality as previously described by Chokeshaiusaha et al. [15].
Nucleotide sequences of annotated non-coding RNAs applied in this study were retrieved from the Ensembl database (https://asia.ensembl.org/info/data/ftp/index. $\mathrm{html})$. Quality trimming and adapter trimming were performed by Cutadapt v1.14 program [16]. Only sequencing reads with length between 18 and 30 nucleotides and having Phred score $\geq 20$ for at least $50 \%$ bases were selected for genome alignment. Alignment was performed with the selected reads corresponding to the species' genomes - UMD3.1 for cow and GRCh38 for human. Any sequence reads aligned to rRNA, tRNA, snRNA, snoRNA genes, or acquired more than five positions aligned were discarded. The leftover aligned reads were subsequently aligned to miRNA genes. The duplicated sequences were removed [17] and corrected for GC base bias [18]. HTSeq package (version 0.9.1) was applied for miRNA counts [15], and regularized logarithm transformations were performed with the count datasets [19].

\section{miRNA target genes and gene-annotation enrich- ment analysis}

High-confidence miRNA target genes were selected according to their cumulative weighted context scores of the sites (context score $\leq 0.4$ and percentile $\geq 85$ ), or aggregate probability of conserved targeting $\geq 0.8$ from TargetScanHuman (release 7.1). Enrichment analysis was subsequently performed using target gene list acquired from each species, and significant gene ontology (GO) terms were identified (adjusted $\mathrm{p} \leq 0.01$ ) using "clusterprofiler" package [20]. The immune-related GO terms were extracted and observed (Figure-1). miRNA target genes presented in these immune-related GO terms were then determined for the number of miRNA genes targeting them. The genes with more than 50 different miRNA genes acquired from each of species were selected (Table-2) and reviewed for their immunological functions.

\section{Data virtualization}

Heatmap of non-scaled and scaled normalized miRNA read count data was drawn by

Table-1: Milk miRNA-seq datasets used in this study.

\begin{tabular}{llc}
\hline Species & Description & Sample files \\
\hline Bos taurus (Dairy cow) & Dataset of milk lipid miRNome sequencing & SRR4030778; SRR4030787; SRR4030792 \\
& approximately at 2 months of lactation & SRR4030797; SRR4030834; SRR4030843 \\
& & SRR4030852; SRR4030860; SRR4030867 \\
& Dataset of milk lipid miRNome sequencing & SRR4030771; SRR4030779; SRR4030788 \\
& approximately at 4 months of lactation & SRR4030798; SRR4030831; SRR4030835 \\
& & SRR4030844; SRR4030861 \\
& Dataset of milk lipid miRNome sequencing & SRR4030772; SRR4030780; SRR4030789 \\
& approximately at 6 months of lactation & SRR4030799; SRR4030836; SRR4030842 \\
& & SRR4030845; SRR4030854; SRR4030862 \\
& & \\
& & \\
Homo sapiens (nursing mother) & Dataset of milk lipid miRNome sequencing & SRR2976292; SRR2976301; SRR2976310 \\
& approximately at 2 months of lactation & SRR2976316; SRR2976322 \\
& Dataset of milk lipid miRNome sequencing & SRR2976293; SRR2976302; SRR2976311 \\
& approximately at 4 months of lactation & SRR2976317; SRR2976323 \\
& Dataset of milk lipid miRNome sequencing & SRR2976294; SRR2976303; SRR2976312 \\
& approximately at 6 months of lactation & SRR2976318; SRR2976324
\end{tabular}

miRNA=MicroRNAs 


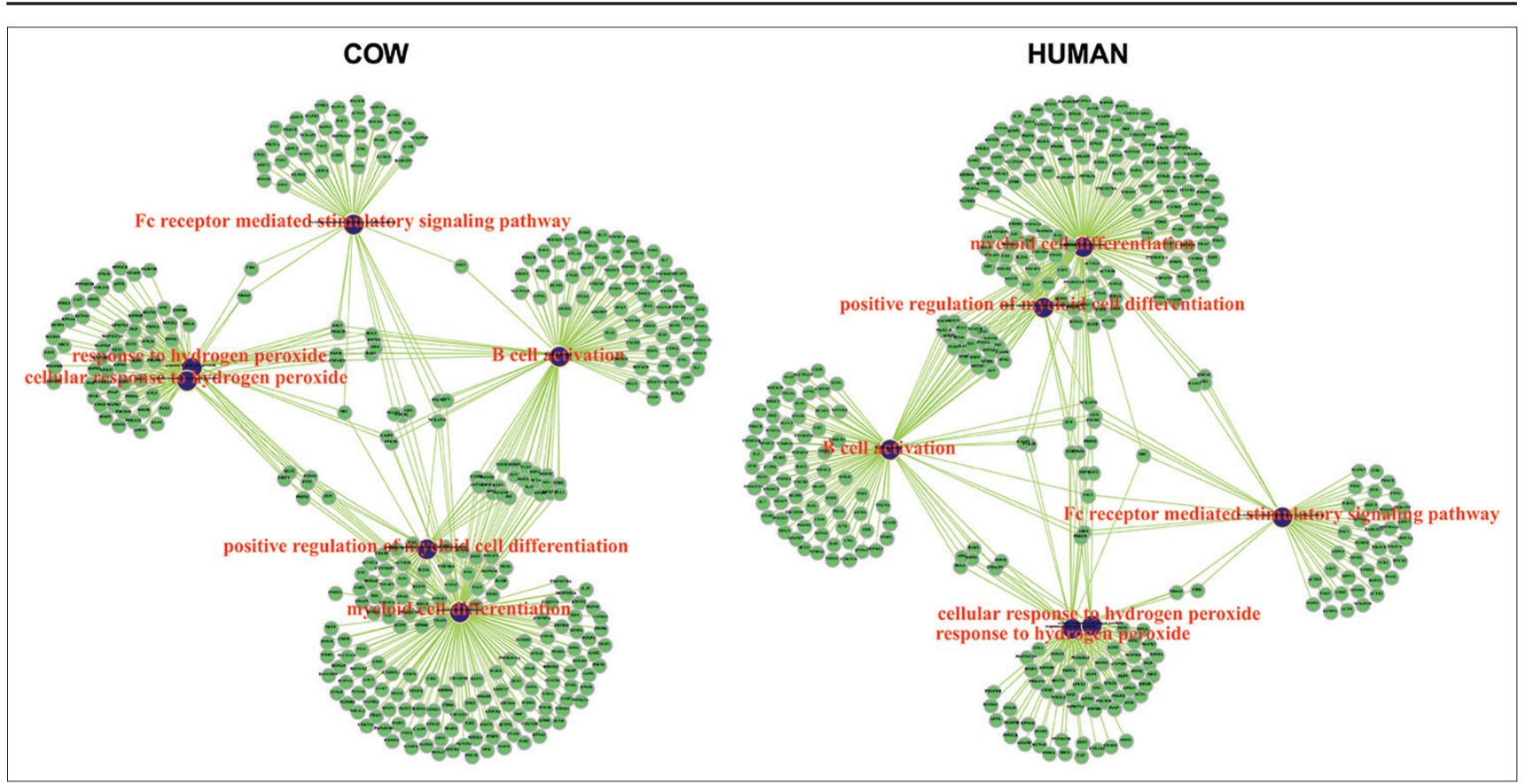

Figure-1: Networks of immune-related gene ontology (GO) terms $(\bullet)$ and target genes of milk microRNAs $(\bullet)$ of cow and human. The presented GO terms were as follows: Response to hydrogen peroxide, myeloid cell differentiation, Fc receptor mediated stimulatory signaling pathway, cellular response to hydrogen peroxide, positive regulation of myeloid cell differentiation, and B-cell activation.

Table-2: Selected immune-associated genes targeted by cow and human milk miRNAs.

\begin{tabular}{|c|c|c|c|c|}
\hline \multirow[t]{2}{*}{ Gene name } & \multirow[t]{2}{*}{ Human Ensembl ID } & \multirow[t]{2}{*}{ Cow Ensembl ID } & \multicolumn{2}{|c|}{$\begin{array}{c}\text { Number of miRNA genes } \\
\text { targeting }\end{array}$} \\
\hline & & & Cow & Human \\
\hline TFRC & ENSG00000072274 & ENSBTAG00000032719 & 60 & 70 \\
\hline HIF1A & ENSG00000100644 & ENSBTAG00000020935 & 60 & 68 \\
\hline CREB1 & ENSG00000118260 & ENSBTAG00000005474 & 68 & 100 \\
\hline MYB & ENSG00000118513 & ENSBTAG00000012074 & 52 & 56 \\
\hline INHBA & ENSG00000122641 & ENSBTAG00000002912 & 93 & 132 \\
\hline ETS1 & ENSG00000134954 & ENSBTAG00000002341 & 172 & 144 \\
\hline KLF4 & ENSG00000136826 & ENSBTAG00000020355 & 76 & 78 \\
\hline PIK3R1 & ENSG00000145675 & ENSBTAG00000010989 & 93 & 96 \\
\hline PURB & ENSG00000146676 & ENSBTAG00000046625 & 51 & 63 \\
\hline KLF10 & ENSG00000155090 & ENSBTAG00000014396 & 50 & 68 \\
\hline PPARGC1B & ENSG00000155846 & ENSBTAG00000012943 & 76 & 126 \\
\hline CRK & ENSG00000167193 & ENSBTAG00000005665 & 110 & 128 \\
\hline $\mathrm{BCL} 2$ & ENSG00000171791 & ENSBTAG00000019302 & 84 & 96 \\
\hline SP3 & ENSG00000172845 & ENSBTAG00000000176 & 50 & 90 \\
\hline
\end{tabular}

miRNA=MicroRNAs

“ComplexHeatmap" package [21] to display miRNAs presented in cow and human milk datasets (Figure-2). To visualize association among the immune-related GO terms and their identified target genes, the network among terms and target genes was plotted using "igraph" package [22] (Figure-1). To illustrate the selection of immune-associated genes targeted by a number of different miRNA genes, the spherical interacting network among them was plotted (Figure-3).

\section{Results and Discussion}

\section{Cow and human milk miRNAs}

To acquire the results that represented mammary gland functions in miRNA productions during mid-lactation period of both cow and human, this study adopted milk lipid miRNome expression profiles during 3-6 months of lactational periods acquired from both species. A total of 146 and 129 miRNAs were identified in cow and human milk, accordingly. Supporting the previous study [2], similar miRNA expressions among three lactation periods were demonstrated (non-scaled heat map of Figure-2). Top 50 expressed miRNAs ranked by mean expression values were presented (Table-3), by which were concordant with other previous reports $[1,6,10]$. Interestingly, some miRNAs with immunoregulatory functions were also included (Table-3). For example, bta-mir-142 (ENSBTAG00000029982) [23], bta-mir-146a (ENSBTAG00000029830) [24], and bta-mir-148b (ENSBTAG00000029852) [25] were 


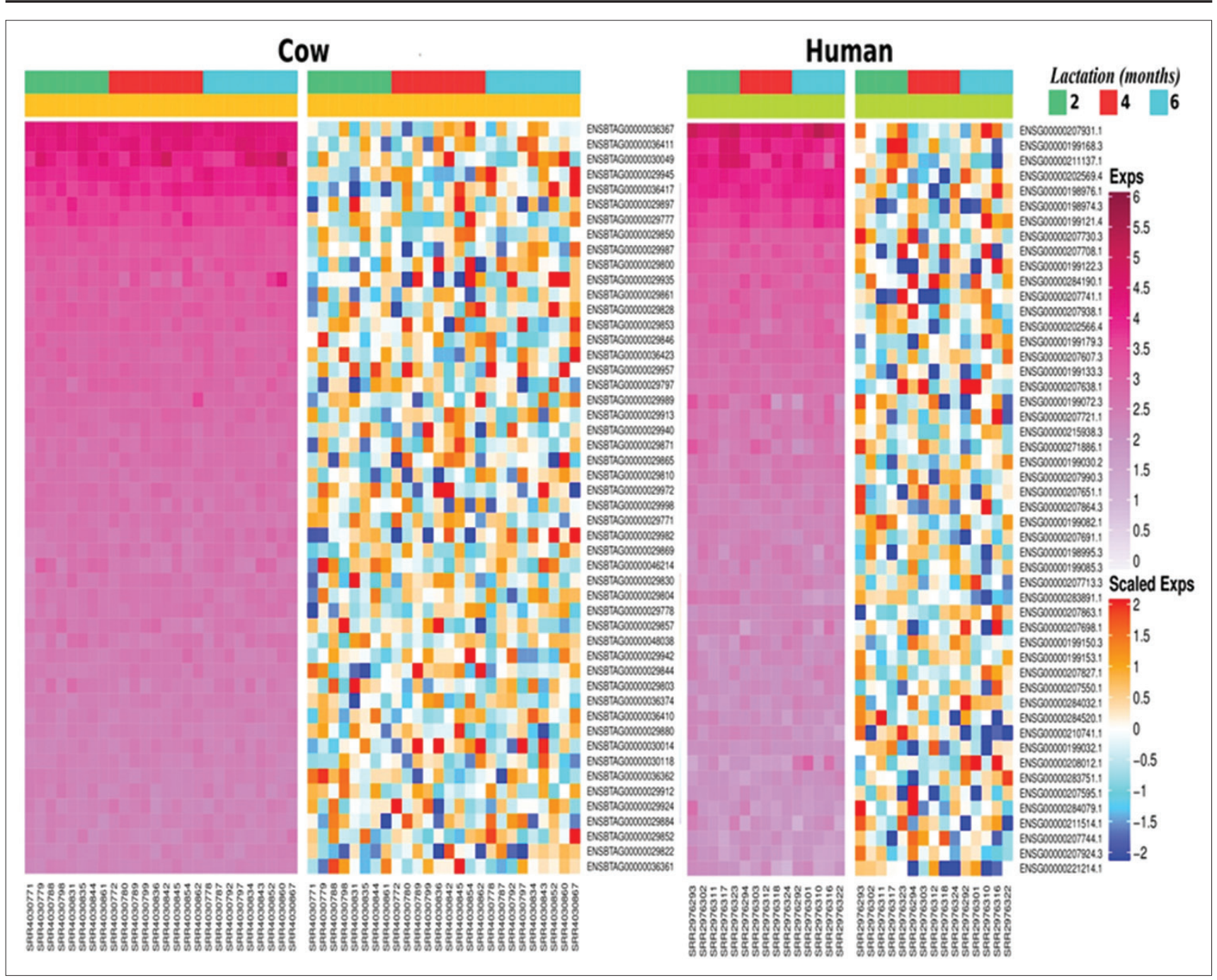

Figure-2: Heatmaps of top 50 cow (cow) and human (human) milk microRNAs read count number in non-scaled regularized logarithm (exps) and scaled regularized logarithm (scaled exps) at $2(\square), 4(\square)$, and $6(\square)$ months of lactation ordering by mean expression values.

evidencedfortheirimmunosuppressivefunctionsincow. Similarly, Hsa-mir-181a-2 (ENSG00000207595.1), Hsa-mir-125b-2 (ENSG00000207863.1), Hsa-mir146b (ENSG00000202569.4), and Hsa-let-7i were well-recognized members of immunosuppressive miRNAs in human [26-29].

\section{Enrichment analysis and prediction of mutual immune-associated target genes of milk miRNAs}

Prediction of milk miRNA target genes was performed by TargetScanHuman. We considered TargetScanHuman suitable for our study due to its principle in comparison of conserved 3'UTR among orthologous sequences of human and other mammalian transcripts including cow [14,30]. All predicted target genes were authorized for gene-annotation enrichment analysis, and several terms associated developmental processes and cell mechanisms were signified (adjusted $\mathrm{p} \leq 0.01$ ) concordant with other previous reports $[1,2]$. Among them, nine terms were biologically immune-related and also shared between cow and human - implying them as the conserved miRNAs regulating processes. These terms were as follows: Response to hydrogen peroxide, myeloid cell differentiation, Fc receptor mediated stimulatory signaling pathway, cellular response to hydrogen peroxide, positive regulation of myeloid cell differentiation, and B-cell activation (Figure-1). The genes related to these terms were then determined for cow and human miRNAs targeting them. The ratio of cow to human miRNAs targeting each gene revealed that most genes were targeted by cow more than human miRNAs (Cow: Human miRNA ratio $>1$ in Figure-3). Among them, 14 genes were featured with more than 50 miRNA genes of each species targeting them (Figure-3 and Table-2) including the top expressed ones (Table-1).

As mentioned in the introduction, lymphocytes were implied as common immune cells affected by intake milk miRNA [6,8-12]. Concordant with other previous reports [6,9-11], our acquired mutual immune-associated genes also manifested their roles with lymphocyte differentiation, activation, and proliferation. Post-transcriptional gene silencing of milk miRNAs on them should thus contribute to modulation of lymphocyte development and also maintaining a non-activating state of lymphocytes of which 

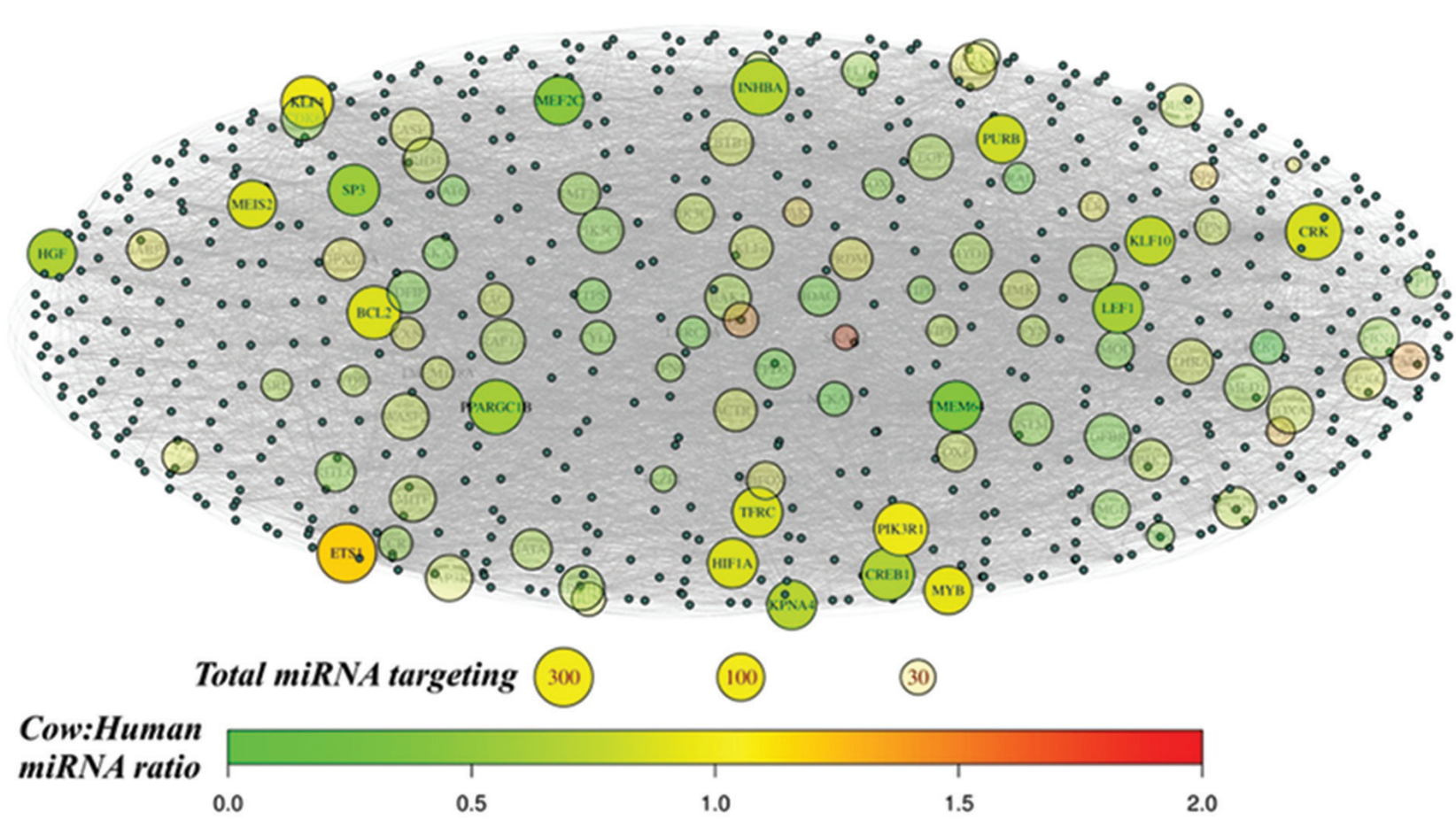

Figure-3: Spherical network of milk microRNAs (miRNA) target genes presented in immune-related gene ontology (GO) terms. Target genes of milk miRNA presented in immune-related GO terms were illustrated in the figure by the number of miRNA targeting them (Total miRNA targeting) indicating by circle size, and also the ratio of cow to human miRNA targeting them scaling from green to red colors (cow: Human miRNA ratio). The target genes with $<50$ miRNA genes acquired from each of species targeting them ( $<50$ cow miRNAs and $<50$ human miRNAs) were transparent and were not reviewed for their functions. Each small gray dots represented miRNA without label.

would be reviewed later. While most previous studies attributed regulatory contributions of milk miRNAs to some well-characterized miRNAs, such assumptions could overlook several other genes significantly targeted by the whole redundant miRNAs presented in milk. In this study, prediction of candidate genes iteratively targeted by overall miRNAs ( $\geq 50$ miRNAs of each species) should provide an additional strategy to determine the immunoregulatory function of milk miRNAs on such genes.

\section{Immune-associated genes iteratively targeted by cow and human milk miRNAs}

Immunoregulatory functions of the acquired 14 genes (Table-2) were briefly reviewed as follows. Expressions of CREB, EST1, KLF4 MYB, PPARGC1B, and TFRC were related to increases in lymphocyte activation, lymphocyte proliferation, and inflammation [31-38]. PURB encoded a transcription activator of MYC molecule - a key transcription factor required during dendritic cell (DC) maturation and lymphocyte activation [39]. HIF1A - a subunit gene of HIF-1 transcription factor could activate DC and inhibit the regulatory function of T-cell simultaneously [40]. INHBA encoded a subunit of activin - a protein required for several pro-inflammatory cytokine releases [41]. PIK3R1 encoded the regulatory subunit 1 of PI3K - a kinase adapter protein required for lymphoid/ myeloid cell development and functions [42]. CRK encodes an adapter protein participating in several cell signaling pathways, while BCL2 encoded a universal antiapoptotic molecule commonly upregulated during lymphocyte development and activation [43,44]. SP3 was crucial for expressions of some surface molecules and cytokines in lymphocytes and myeloid cells $[45,46]$. Among all predicted target genes, KLF10 was the only gene with a suppressive function on T-cell proliferation $[47,48]$. While the effective evaluation system for overall milk miRNAs' regulation on target leukocyte was still undetermined, these target genes could become potential candidates for such studies in the future.

\section{Conclusion}

The current study demonstrated a novel procedure to determine immunoregulatory genes comprehensively targeted by cow and human milk miRNAs. The acquired candidate genes - based on immune-related GO terms strongly suggested the plausible roles of milk miRNAs in maintaining the cell's homeostasis and its resting state. With validated determination system, these candidate genes should hereby establish numbers of further interesting novel research topics. Of note, this study aimed to demonstrate a beneficial strategy for future cross-species miRNA target genes employing immune system for demonstration - by which was able to apply with other biological systems of interest.

\section{Authors' Contributions}

$\mathrm{KC}$ and TS planned the study design, collected the datasets and analyzed the data. $\mathrm{CN}$ refined the study design and the objective. DP carried out technical 
Table-3: Top 50 miRNA genes presented in cow and human milk.

\section{Organisms Top 50 miRNAs}

Cow ENSBTAG00000036367, ENSBTAG00000036411, ENSBTAG00000030049,

ENSBTAG00000029945, ENSBTAG00000036417, ENSBTAG00000029897, ENSBTAG00000029777,

ENSBTAG00000029850, ENSBTAG00000029987 ENSBTAG00000029800, ENSBTAG00000029935, ENSBTAG00000029861,

ENSBTAG00000029828, ENSBTAG00000029853, ENSBTAG00000029846,

ENSBTAG00000036423,

ENSBTAG00000029957, ENSBTAG00000029797 ENSBTAG00000029989, ENSBTAG00000029913, ENSBTAG00000029940,

ENSBTAG00000029871,

ENSBTAG00000029865, ENSBTAG00000029810

ENSBTAG00000029972,

ENSBTAG00000029998，ENSBTAG00000029771

ENSBTAG00000029982,

ENSBTAG00000029869, ENSBTAG00000046214

ENSBTAG00000029830,

ENSBTAG00000029804, ENSBTAG00000029778

ENSBTAG00000029857,

ENSBTAG00000048038，ENSBTAG00000029942

ENSBTAG00000029844,

ENSBTAG00000029803，ENSBTAG00000036374

ENSBTAG00000036410,

ENSBTAG00000029880, ENSBTAG00000030014 ENSBTAG00000030118, ENSBTAG00000036362, ENSBTAG00000029912,

ENSBTAG00000029924,

ENSBTAG00000029884, ENSBTAG00000029852 ENSBTAG00000029822, ENSBTAG00000036361

Human ENSG00000207931.1, ENSG00000199168.3, ENSG00000211137.1

ENSG00000202569.4, ENSG00000198976.1, ENSG00000198974.3

ENSG00000199121.4, ENSG00000207730.3, ENSG00000207708.1

ENSG00000199122.3, ENSG00000284190.1, ENSG00000207741.1

ENSG00000207938.1, ENSG00000202566.4, ENSG00000199179.3

ENSG00000207607.3, ENSG00000199133.3, ENSG00000207638.1

ENSG00000199072.3, ENSG00000207721.1, ENSG00000215938.3

ENSG00000271886.1, ENSG00000199030.2, ENSG00000207990.3

ENSG00000207651.1, ENSG00000207864.3, ENSG00000199082.1

ENSG00000207691.1, ENSG00000198995.3, ENSG00000199085.3

ENSG00000207713.3, ENSG00000283891.1， ENSG00000207863.1

ENSG00000207698.1, ENSG00000199150.3， ENSG00000199153.1

ENSG00000207827.1, ENSG00000207550.1, ENSG00000284032.1

ENSG00000284520.1, ENSG00000210741.1, ENSG00000199032.1

ENSG00000208012.1, ENSG00000283751.1, ENSG00000207595.1

ENSG00000284079.1, ENSG00000211514.1, ENSG00000207744.1

ENSG00000207924.3, ENSG00000221214.1

miRNA $=$ MicroRNAs coding correction and hardware maintenance. $\mathrm{KS}$ and TS rafted and reviewed the manuscript. All authors read and approved the final manuscript.

\section{Acknowledgments}

The current study was partially supported by Thailand Research Fund (TRF) through New Research Scholar Program (Grant No. TRG5880003).

\section{Competing Interests}

The authors declare that they have no competing interests.

\section{References}

1. Li, R., Dudemaine, P.L., Zhao, X., Lei, C. and IbeaghaAwemu, E.M. (2016) Comparative analysis of the miRNome of bovine milk fat, whey and cells. PLoS One, 11(4): e0154129.

2. Alsaweed, M., Lai, C.T., Hartmann, P.E., Geddes, D.T. and Kakulas, F. (2016) Human milk cells and lipids conserve numerous known and novel miRNAs, some of which are differentially expressed during lactation. PLoS One, 11(4): $\mathrm{e} 0152610$.

3. Chen, T., Xi, Q.Y., Ye, R.S., Cheng, X., Qi, Q.E. and Wang, S.B.I. (2014) Exploration of microRNAs in porcine milk exosomes. BMC Genomics, 15(1): e1471-2164-15-100.

4. Izumi, H., Kosaka, N., Shimizu, T., Sekine, K., Ochiya, T. and Takase M. (2014) Time-dependent expression profiles of microRNAs and mRNAs in rat milk whey. PLoS One, 9(2): e88843

5. Modepalli, V., Kumar, A., Hinds, L.A., Sharp, J.A., Nicholas, K.R. and Lefevre, C. (2014) Differential temporal expression of milk miRNA during the lactation cycle of the marsupial tammar wallaby (Macropus eugenii). BMC Genomics, 15(1): e1471-2164-15-1012.

6. Kosaka, N., Izumi, H., Sekine, K. and Ochiya, T. (2010) MicroRNA as a new immune-regulatory agent in breast milk. Silence, 1(1): 7.

7. Winter, J., Jung, S., Keller, S., Gregory, R.I. and Diederichs, S. (2009) Many roads to maturity: MicroRNA biogenesis pathways and their regulation. Nat. Cell Biol., 11(3): 228-234.

8. Izumi, H., Tsuda, M., Sato, Y., Kosaka, N., Ochiya, T. and Iwamoto, H.I.W. (2015) Bovine milk exosomes contain microRNA and mRNA and are taken up by human macrophages. J. Dairy Sci., 98(5): 2920-2933.

9. Baier, S.R., Nguyen, C., Xie, F., Wood, J.R. and Zempleni, J. (2014) MicroRNAs are absorbed in biologically meaningful amounts from nutritionally relevant doses of cow milk and affect gene expression in peripheral blood mononuclear cells, HEK-293 kidney cell cultures, and mouse livers. J. Nutr., 144(10): 1495-1500.

10. Sun, Q., Chen, X., Yu, J., Zen, K., Zhang, C.Y. and Li, L. (2013) Immune modulatory function of abundant immune-related microRNAs in microvesicles from bovine colostrum. Protein Cell, 4(3): 197-210.

11. Admyre, C., Johansson, S.M., Qazi, K.R., Filen, J.J., Lahesmaa, R. and Norman, M.I.W. (2007) Exosomes with immune modulatory features are present in human breast milk. J. Immunol., 179(3): 1969-1978.

12. Li, Q.J., Chau, J., Ebert, P.J.R., Sylvester, G., Min, H. and Liu, G.I.W. (2007) miR-181a is an intrinsic modulator of T cell sensitivity and selection. Cell, 129(1): 147-161.

13. Gaidatzis, D., Nimwegen, E., van Hausser, J. and Zavolan, M. (2007) Inference of miRNA targets using evolutionary conservation and pathway analysis. $B M C$ Bioinformatics, 8: 69. 
14. Friedman, R.C., Farh, K.K.H., Burge, C.B. and Bartel, D.P. (2009) Most mammalian mRNAs are conserved targets of microRNAs. Genome Res., 19(1): 92-105.

15. Chokeshaiusaha, K., Thanawongnuwech, R., Puthier, D. and Nguyen, C. (2016) Inspection of C-type lectin superfamily expression profile in chicken and mouse dendritic cells. Thai. J. Vet. Med., 46(3): 443-453.

16. Martin, M. (2011) Cutadapt removes adapter sequences from high-throughput sequencing reads. EMBnet J., 17(1): 10.

17. Li, H., Handsaker, B., Wysoker, A., Fennell, T., Ruan, J. and Homer, N.I.W. (2009) The sequence alignment/map format and SAM tools. Bioinformatics, 25(16): 2078-2079.

18. Ramírez, F., Dündar, F., Diehl, S., Grüning, B.A. and Manke, T. (2014) Deep tools: A flexible platform for exploring deep-sequencing data. Nucleic Acids Res., 42(W1): W187-W191.

19. Love, M.I., Anders, S. and Huber, W. (2014) Differential analysis of count data-the DESeq2 package. Genome Biol., 15: 550 .

20. Yu, G., Wang, L.G, Han, Y. and He, Q.Y. (2012) Cluster profiler: An R package for comparing biological themes among gene clusters. Omi A J. Integr. Biol., 16(5): 284-287.

21. Gu, Z., Eils, R. and Schlesner, M. (2016) Complex heatmaps reveal patterns and correlations in multidimensional genomic data. Bioinformatics, 32(18): 2847-2849.

22. Csárdi, G. and Nepusz, T. (2006) The igraph software package for complex network research. Int. J. Complex Syst., 1695: 1-9.

23. Wang, X.P., Luoreng, Z.M., Zan, L.S, Li, F. and Li, N. (2017) Bovine miR-146a regulates inflammatory cytokines of bovine mammary epithelial cells via targeting the TRAF6 gene. J. Dairy Sci., 100(9): 7648-7658.

24. Sonda, N., Simonato, F., Peranzoni, E., Calì, B., Bortoluzzi, S. and Bisognin, A.I.W. (2013) MiR-142-3p prevents macrophage differentiation during cancer-induced myelopoiesis. Immunity, 38(6): 1236-1249.

25. Wu, H., Jiang, K., Ma, X., Yin, N., Zhao, G. and Qiu, C.I.W. (2018) IFN- $\tau$ mediated control of bovine major histocompatibility complex class I expression and function via the regulation of bta-miR-148b/152 in bovine endometrial epithelial cells. Front Immunol., 9: 167.

26. Zhu, J., Yao, K., Guo, J., Shi, H., Ma, L. and Wang, Q.I.W. (2017) miR-181a and miR-150 regulate dendritic cell immune inflammatory responses and cardiomyocyte apoptosis via targeting JAK1-STAT1/c-Fos pathway. J. Cell Mol. Med., 21(11): 2884-2895.

27. Lee, H.M., Kim, T.S. and Jo, E.K. (2016) MiR-146 and miR-125 in the regulation of innate immunity and inflammation. BMB Rep., 49(6): 311-318.

28. Sathe, A., Ayyar, K. and Reddy, K.V.R. (2014) MicroRNA let-7 in the spotlight: Role in innate immunity. Inflamm. Cell Signal, 1: 66-75.

29. Chen, X.M., Splinter, P.L., O'Hara, S.P. and La Russo, N.F. (2007) A cellular micro-RNA, let-7i, regulates toll-like receptor 4 expression and contributes to cholangiocyte immune responses against Cryptosporidium parvum infection. J. Biol. Chem., 282(39): 28929-28938.

30. Witkos, T.M., Koscianska, E. and Krzyzosiak, W.J. (2011) Practical aspects of microRNA target prediction. Curr. Mol. Med., 11(2): 93-109.

31. Raghunandan, R., Frissora, F.W. and Muthusamy, N. (2013) Modulation of Ets-1 expression in B lymphocytes is dependent on the antigen receptor-mediated activation signals and cell cycle status. Scand. J. Immunol., 77(2): 75-83.
32. Garrett-Sinha, L.A. (2013) Review of Ets1 structure, function, and roles in immunity. Cell Mol. Life Sci., 70(18): 3375-3390.

33. Klaewsongkram, J., Yang, Y., Golech, S., Katz, J., Kaestner, K.H. and Weng, N.P. (2007) Krüppel-like factor 4 regulates $\mathrm{B}$ cell number and activation-induced $\mathrm{B}$ cell proliferation. J. Immunol., 179(7): 4679-4684.

34. Hart, G.T., Hogquist, K.A. and Jameson, S.C. (2012) Krüppel-like factors in lymphocyte biology. J. Immunol., 188(2): 521-526.

35. Wen, A.Y., Sakamoto, K.M. and Miller, L.S. (2010) The role of the transcription factor CREB in immune function. J. Immunol., 185(11): 6413-6419.

36. Preston, G.C., Sinclair, L.V., Kaskar, A., Hukelmann, J.L., Navarro, M.N. and Ferrero, I.I.W. (2015) Single cell tuning of Myc expression by antigen receptor signal strength and interleukin-2 in T lymphocytes. EMBO J., 34(15): 2008-2024.

37. Donnell, K.A.O., Yu, D., Zeller, K.I., Kim, J., Racke, F. and Dang, C.V.I. (2006) Activation of transferrin receptor 1 by c-Myc enhances cellular proliferation and tumorigenesis activation of transferrin receptor 1 by c-Myc enhances cellular proliferation and tumorigenesis. Mol. Cell Biol., 26(6): 2373-2386

38. Sweeney, T.E., Suliman, H.B., Hollingsworth, J.W. and Piantadosi, C.A. (2010) Differential regulation of the PGC family of genes in a mouse model of Staphylococcus aureus sepsis. PLoS One, 5(7): e11606.

39. Gnanaprakasam, J.N.R. and Wang, R. (2017) MYC in regulating immunity: Metabolism and beyond. Genes (Basel), 8(3): 88 .

40. Kumar, V. and Gabrilovich, D.I. (2014) Hypoxia-inducible factors in regulation of immune responses in tumour microenvironment. Immunology, 143(4): 512-519.

41. Xia, Y. and Schneyer, A.L. (2009) The biology of activin: Recent advances in structure, regulation and function. J. Endocrinol., 202(1): 1-12.

42. Okkenhaug, K. (2013) Signaling by the phosphoinositide 3-kinase family in immune cells. Annu. Rev. Immunol., 31(1): 675-704.

43. Renault, T.T. and Chipuk, J.E. (2013) Getting away with murder: How does the BCL-2 family of proteins kill with immunity? Ann. N. Y. Acad. Sci., 1285(1): 59-79.

44. Broome, H.E, Dargan, C.M., Krajewski, S. and Reed, J.C. (1995) Expression of Bcl-2, Bcl-x, and Bax after T cell activation and IL-2 withdrawal. J. Immunol. (Baltimore, $M d$ 1950), 155(5): 2311-2317.

45. Noti, J.D. (1977) Sp3 mediates transcriptional activation of the leukocyte integrin genes $\mathrm{CD} 11 \mathrm{C}$ and $\mathrm{CD} 11 \mathrm{~B}$ and cooperates with c-Jun to activate CD11C. J. Biol. Chem., 272(38): 24038-24045.

46. Grekova, M.C., Salerno, K., Mikkilineni, R. and Richert, J.R. (2002) Sp3 expression in immune cells: A quantitative study. Lab Investig., 82(9): 1131-1138.

47. Cao, Z., Wara, A.K., Icli, B., Sun, X., Packard, R.R.S. and Esen, F.I.W. (2009) Kruppel-like factor KLF10 targets transforming growth factor-betal to regulate $\mathrm{CD} 4(+)$ CD25(-) T cells and T regulatory cells. J. Biol. Chem., 284(37): 24914-24924.

48. Papadakis, K.A., Krempski, J., Reiter, J., Svingen, P., Xiong, Y. and Sarmento, O.F.I. (2015) Krüppel-like factor KLF10 regulates transforming growth factor receptor II expression and TGF- $\beta$ signaling in CD8+ T lymphocytes. Am. J. Physiol. Cell. Physiol., 308(5): C362-C371. 